\title{
Non-conventional caries management techniques for children show promise
}

\author{
Abstracted from \\ Santamaria RM, Innes NP, Machiulskiene V, Evans DJ, Alkilzy M, Splieth CH.
}

Acceptability of different caries management methods for primary molars in a RCT. Int J Paediatr Dent 2014; Mar 7. doi: 10.1111/ipd.12097. [Epub ahead of print] PubMed

Address for correspondence: Ruth M. Santamaria, Department of Preventive and Pediatric Dentistry, Ernst-Moritz-Arndt University of Greifswald, Rotgerberstr. 8, 17487 Greifswald, Germany. E-mail: ruth.santamaria@uni-greifswald.de

\section{Question: In children with approximal dentinal caries lesions in primary molars do different treatment strategies affect behaviour and pain perception?}

Design Secondary care-based, three-arm parallel-group, randomised controlled trial.

Intervention Children aged three to eight years old with at least one primary molar tooth with caries into dentine involving two dental surfaces were randomised to either; conventional restoration (CR) with local anaesthesia, Hall technique (HT) or non-restorative caries treatment (NRCT) with no local anaesthetic. Only one tooth per child was included and parents were present during treatment.

Outcome measure Child's pain perception (Visual Analogue Scale of Faces); behaviour (Frankl scale); and parents' and dentists' treatment opinions (five-point Likert scales).

Results One hundred and sixty-nine children (96 boys and 73 girls) were randomised; 52 received HT; $52 \mathrm{NRCT}$ and $65 \mathrm{CR}$. Children showed more negative behaviour in the $\mathrm{CR}$ group (37\%) compared to NRCT (21\%) and HT (13\%) (P=0.047, Cl= 0.41 to 0.52$)$. Pain intensity was rated 'very low' or 'low' in $88 \%$ NRCT, $81 \% \mathrm{HT}$, and $72 \%$ $\mathrm{CR}(\mathrm{P}=0.11, \mathrm{Cl}=0.10$ to 0.12$)$. NRCT and $\mathrm{HT}$ were 'very easy' or 'easy' to perform for $>77 \%$ of dentists, compared to $50 \%$ in $C R$ group $(\mathrm{P}<$ $0.000)$. There were no statistically significant differences in parents' rating of their child's level of comfort ( $\mathrm{P}=0.46, \mathrm{Cl}=0.45$ to 0.48 ). Conclusions Dentists reported more negative behaviour in the $\mathrm{CR}$ group. For all techniques, children's pain perception and dentist/parent acceptability were similar..

\section{Commentary}

Dental caries is the most common childhood disease and noncommunicable disease worldwide ${ }^{1} \mathrm{~A}$ concerning but unsurprising sequel of the severity and prevalence of dental disease is that it is the fourth most common reason why a child is admitted to hospital in England. ${ }^{2}$ This study addresses a valid, under-reported and current predicament facing many dental professionals, across primary and secondary care. Dentists often struggle to decide on the best treatment option that encompasses operator preference, effectiveness and specifically acceptability to patient and parents.
There are many studies into the success of various treatment modalities for carious deciduous teeth, and guidelines have been published by SDCEP $^{3}$ and BSPD $^{4}$ but this study appears to be the first RCT to address the acceptance and perception of three treatment options. Furthermore as there is a growing interest in the conservative, atraumatic minimal invasive approach, this study investigates its acceptability amongst dentists, patients and parents.

The study being an RCT gave hierarchical design value but it also involved a qualitative data collection. The study addresses a clearly focused issue with measurable outcomes. Children's behaviour and pain perception were measured when approximal dentinal caries lesions in primary molars (in children aged three to eight years) were managed with three treatment strategies; conventional restorations (CR), Hall technique (HT), and non-restorative caries treatment (NRCT). The acceptability of the techniques to parents and dentists was also investigated.

The assignment of patients was sequentially randomised, using a computer-generated random number list with allocation concealment, to one of three arms. This randomisation of patients will strengthen the validity of the statistical tests used. However the authors have not made it clear whether the dentists themselves were randomised to the treatment group. If dentists were not randomised, this would have certainly introduced pre-trial bias that could have impacted on the results of the study as dentists' acceptability and their delivery of treatment would differ, in turn, which may inadvertently affect a child's behaviour and perception.

A single researcher recruited the patients following inclusion and exclusion criteria. But this requires some clarification as the paper also states 'The 12 participating dentists recruited between one and 40 patients over a period of 18 months'. This raises some confusion as to whether a single researcher was solely involved in recruitment of all participants. It is not clear if the dentists followed the inclusion/exclusion criteria or whether they were involved in just selecting the eligible patients. Either way there is a risk of selection bias.

The study makes no reference to how the sample size was calculated, which would have been helpful.

The paper reports that the study was discussed with parents and participants and written consent was obtained, although there is no detail of the information shared. There may be a risk of bias, particularly if there was a chance that parents were aware of the aim of the study. It cannot be confirmed that participants and parents were 'blind' to this. The child's perception to treatment may 
be altered to provide answers in order to please the clinician or what they consider to be socially acceptable (which will, for example, influence their response to the five-point likert scale).

Outcome measures were immediately carried out at the same appointment by the dentist. Unfortunately this approach opens itself to a variety of bias. There may be consistency bias as all three outcomes were measured one after the other and patients'/parents' previous responses may influence their next one.

Results may be also be influenced by moderator acceptance bias as both the patients and parents may provide answers to please the dentist. Furthermore patients have just finished treatment and their mood may not allow them to provide a reflective true response. There also could be bias reporting on behalf of the dentists; this could be unconsciously influenced by their experience, beliefs, wishes, attitudes, etc.

As a result of the ambiguity over the integrity of 'blindness', more explicit detail on the consent process and description of masking needs could have been described. The clinicians could not have been blinded to the intervention. As the dentists were aware of the treatment pathway their patients had been allocated to and bearing in mind the aim of study, they could have unconsciously altered their performance.

The study endeavoured to keep the patient groups similar. By utilising groups of subjects with similar characteristics, the randomisation will minimise the confounding bias that occurs when one group of participants have certain features, for example, gender differences in pain perception/threshold. A shortcoming of the study is that there is no reference to social class, ethnicity, social history or past dental history: all of which can affect patient behaviour and pain perception to treatment.

The study was carried in only one university in Germany in a recognised diverse city, however whether the findings are generalisable needs to be considered.

The authors report that the $75 \%$ of the dentists within this study were $26-45$ years of age with $58 \%$ female. There was training on carrying out the intervention as well as calibration on outcome measures. Calibration usually involves a robust procedure and it would have been beneficial if the authors included detail of this, to determine the likelihood of undermining the process.

The authors have endeavoured to increase generalisability of study by including 12 different dentists, they are all, however, either paediatric specialists or post-graduate paediatric students and undoubtedly their technique and skill is likely to be different from other dental professionals.

Most of the clinically important outcomes were considered in this study. However, many aspects of the study were not controlled or monitored and as such there are many unmeasured variables that could have affected the outcome, thus affecting inter-examiner reliability. Therefore to be able to contextualise the findings, it would have been useful to have further information on the following:

- Whether behaviour management techniques were used for example, tell, show, do

- Whether patients had previous non-pharmacological behaviour management, which could have influenced their behaviour and acceptability during the trial

- Method of LA administration (was topical used?)

- Length of procedure
- Objective signs of pain, for example, crying, as there may be bias on dentist's subjective perception of child's experienced pain

The authors reported no significant difference with use of LA and by the dentists' level of experience. However, it can be queried how this could have been established. It may be a presumption made after analysing the results and cannot be considered a direct conclusion.

Moreover, the study doesn't touch on the determinants of acceptability among parents and children. For some parents the thought of any caries left behind will be unacceptable, even though the patient had a good chair-side experience, whereas for others as long as the child is pain free there is no concern over treatment modality. Determinants of acceptability are more or less subjective and therefore can generalised conclusions be drawn from this study?

It perhaps would have been helpful if the study elaborated on this. The authors further comment that if more than one tooth was eligible for treatment, the next tooth on the prescribed treatment plan was chosen by the single researcher for the study. However do dentists really follow a specific tooth-by-tooth treatment plan?

It could be argued that the inclusion criteria could have been more rigorous by just including the first episode of treatment, or new patients to increase external validity. Furthermore decay into dentine needed to involve two surfaces but the inclusion criteria did not dictate which two surfaces as this could influence ease of treatment, moisture control and pain perception.

This study has examined behaviour and discomfort on different treatment modalities, which is rarely reported and thus this study is likely to hold recognition among dental professionals.

\section{Practice points}

- No significant difference in acceptability found between the three treatment modalities.

- Interestingly the study found that the use of LA had no impact on pain perception and acceptability.

- No significant difference found between dentist experience.

- External validity low due to technique sensitivities and population sample.

- Study will yield further clinical value post follow-up into clinical effectiveness of procedure.

\section{Sharaniya Thillainathana and Brett Duane ${ }^{b}$}

${ }^{a}$ Department of Special Care Dentistry and Dental Public Health,

Health Education Kent, Surrey and Sussex,

Crawley Hospital, Crawley, West Sussex

${ }^{b}$ Public Health England, County Hall North, Chart Way, Horsham, West Sussex

1. Oral Health Worldwide. A report by FDI World dental Federation. 2014

2. Hansard. Written answers. Hospitals: admissions (Parliamentary debates 16 April 2012). House of Commons Official Report 2012; 543: 116W Online debates available at http://www.publications. parliament.uk/pa/cm201212/cmhansrd/chan288.pdf (accessed April 2013).

3 Scottish Dental Clinical Effectiveness Programme 2010. Prevention and Management of Dental Caries in Children.

4 Kandiah T, Johnson J, Fayle SA. British Society of Paediatric Dentistry: a policy document on management of caries in the primary dentition. Int J Paediatric Dent 2010; 20: doi: 10.1111/j.1365-263X.2010.01087.x. 